\title{
Making Demand Response a Reality in Europe: Policy, Regulations and Deployment Status
}

\author{
Ilias Lamprinos, Nikos D. Hatziargyriou, Isidoros Kokos, and Aris Dimeas
}

\begin{abstract}
Power systems undergo massive operational and technological changes amid increasing demand for environmental sustainability and energy efficiency. The traditional, supplydriven approach, relying on large-scale generation plants, which has dominated old utilities, is reconsidered to incorporate the increased penetration of variable renewable energy sources, distributed generation and storage. Demand Response is an important instrument for improving energy efficiency, since it increases consumers' engagement and provides a mechanism to reduce or shift consumption, resulting in energy savings. Regulators and policy makers in several European countries take substantial measures to encourage market uptake of Demand Response as a means to mitigate the limitations of the existing grid and boost the transition to a low carbon economy. This goes hand in hand with the deployment of services by stakeholders who aggregate consumption flexibility and offer it to grid operators or to the electricity market. Several large-scale pilot projects explore the feasibility of Demand Response in residential and small commercial customers. This paper provides an overview of the current regulatory and policy framework in Europe and summarizes the state of play of commercial and pilot Demand Response deployments in various European countries. Also, it highlights some key research objectives associated with Demand Response.
\end{abstract}

Index Terms - demand response, smart grid, flexibility, prosumer

\section{INTRODUCTION}

$\mathrm{E}$ urope's energy landscape is experiencing profound change as increasing amounts of Renewable Energy Sources (RES) and other variable generation resources displace conventional forms of generation [1]. This development goes hand-in-hand with an expanding share of power production that takes place at the distribution level. However, variations in wind and solar generation result in a distribution system that is more complex to plan, to control and to balance. The pattern of generation moves away from primarily dispatchable to variable, distributed generation and

This work was supported in part the European Commission's Horizon 2020 Framework Programme (H2020/2014-2020) under Grant Agreement No.646470, SmarterEMC2 Project.

I. Lamprinos and I. Kokos are with Intracom Telecom, GR 19002, Peania, Greece (e-mail: \{labil, isik\}@ intracom-telecom.com).

N. D. Hatziargyriou is with Hellenic Distribution Network Operator, GR 11743, Athens, Greece (e-mail: n.chatziargyriou@ deddie.gr)

A. Dimeas is with Institute of Communications and Computer Systems, GR 15773, Athens, Greece (adimeas@ power.ntua.gr) the share of distributed resources increase significantly. At the same time, the energy consumer becomes also an energy provider (prosumer) and the energy from distributed microgeneration units is fed back to the power grid.

The rising share of distributed generation in the system results in lower predictability for the electricity markets and for the grid operation, and implies the need for increased flexibility to cope with this volatility. Flexibility can help to avoid inefficiency in the markets and allay the concerns that the future mix of generation capacity may not meet demand at optimal costs [2]. For these reasons it is strongly believed that initiatives that facilitate flexibility along with consumer engagement and empowerment will replace or at least defer traditional investments on the grid.

When discussion comes to enablers of the consumer engagement and empowerment, Demand Response (DR) is a potential game-changing concept, which can alter completely the fundamentals of the energy industry. This concept is spread from the energy market layer down to the consumer layer (Fig.1). DR is linked to changes made by end users in terms of their regular energy consumption. It is formed around incentives tailored to shape energy load profiles and serves as a tool to assist the grid operation when reliability is compromised. DR interventions are particularly facilitated by the emergence of intelligence at the edges of the grid and the increasing use of energy management technologies at residential, commercial and industrial buildings.

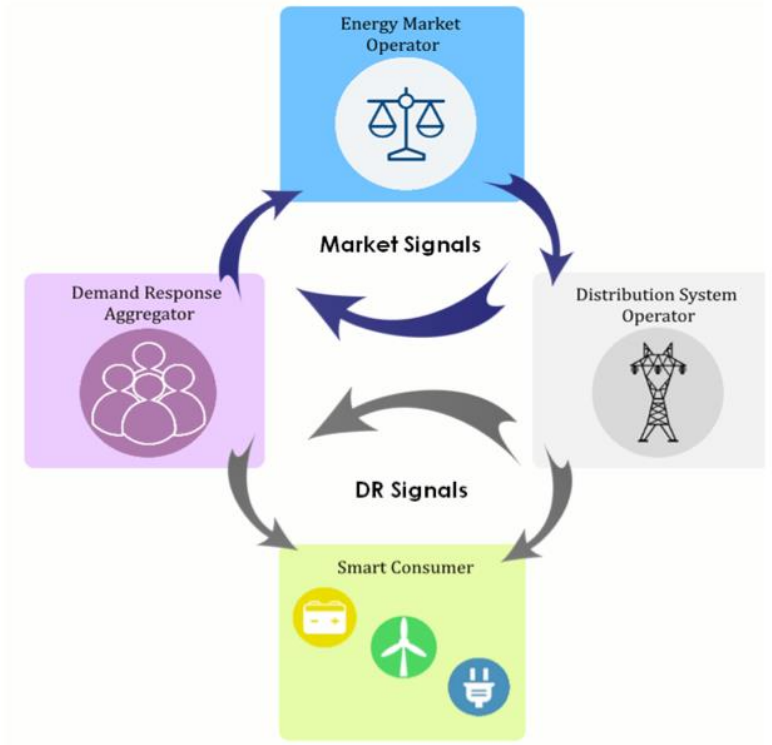

Fig. 1. Demand Response conceptual diagram. 
TABLE I

INDICATIVE DEMAND RESPONSE PROGRAMS

\begin{tabular}{cc}
\hline \hline Implicit DR Programs & Explicit DR Programs \\
\hline Time of Use Pricing & Direct Load Control \\
Critical Peak Pricing & Interruptible Pricing \\
Real Time Pricing & Demand Bidding \\
\hline \hline
\end{tabular}

There are two flavors of DR: explicit and implicit [3]. In explicit DR schemes (also called "incentive-based") consumers receive direct payments to change their consumption upon request (i.e., consuming more or less), which is typically triggered by activation of balancing services, differences in electricity prices or a constraint on the network. Consumers can earn from their flexibility in electricity consumption individually or by contracting with an aggregator. The latter can either be an independent DR Aggregator or the customer's Energy Retailer. Implicit DR (also called "price-based") refers to the cases in which consumers are exposed to time-varying electricity prices or time-varying network tariffs (or both) that partly reflect the value or cost of electricity production and/or transportation in different time periods and react to those price differences depending on their own preferences and constraints. Table I presents indicative implicit and explicit DR Programs. Neither flavor of DR is a replacement of the other. In fact DR Aggregators may leverage different combinations of DR programs with the aim to achieve the desired load curve (Fig.2).

DR offers a novel approach to reconcile and balance generation and consumption and can play a key role in the way consumers interact with the electricity markets either directly or through nominated third parties. Its impact is multifaceted. First, DR is a key tool for grid operators (DSOs, TSOs) to compensate for the intermittency of RES. Second, it is a valuable tool for boosting the transition to a low carbon economy. Third, it provides the energy consumers with financial benefits, either in the form of electricity bill savings or in the form of incentive payments for the consumption flexibility service they offer.

That is why European regulators and policy makers need to take substantial measures to encourage market uptake of DR. In Section II we present an outlook of the current activities in this domain, while Section III provides a high level assessment of the existing European regulatory framework and Section IV summarizes the DR-related standardization activities supported or adopted by European Commission.

Regulation and policy-making related to DR goes hand in hand with the deployment of services by stakeholders who aggregate consumption flexibility and offer it to grid operators or to the electricity market. Until recently however, the typical DR programs in Europe were focused on large industrial consumers, through interruptible tariffs and time-of-use tariffs. A mixture of obstacles related to the regulatory framework, the technology maturity, the electricity markets' design and the offered incentives have strongly limited the participation in DR programs of small customers who make up a plurality of electrical power demand.

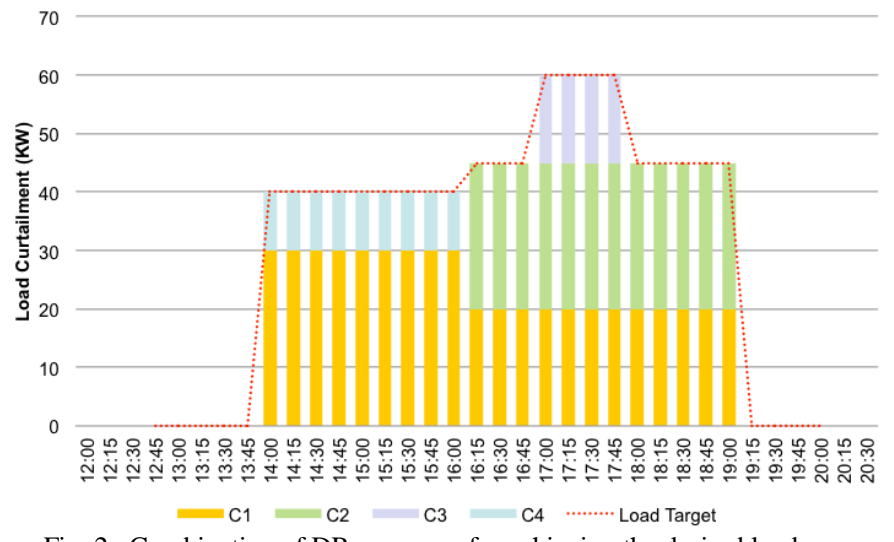

Fig. 2. Combination of DR programs for achieving the desired load curve.

The ongoing activities in the Smart Grids' related European policy, regulation, and standardization affect also the way DR is deployed both as a pilot and as a commercial service. Several ongoing large-scale pilots explore the feasibility of DR in residential and small commercial customers. Section V summarizes the European state of play in commercial deployment of DR services and Section VI presents relevant research and innovation threads co-financed European funds as part of its work programmes. Finally Section VII concludes our paper.

\section{EUROPEAN POLICY RELATED TO THE AdOPTION OF DEMAND RESPONSE}

DR is recognized already in Directive 2009/72/EC [4] regarding common rules for the internal electricity market, as a tool that contributes significantly to enhancing the security of supply and achieving energy efficiency. The roll-out of intelligent metering systems is also promoted (where costeffective), thus facilitating the participation of active electricity customers to the supply market and the application of new forms of electricity tariffs.

Furthermore, as the advantages both for the electricity customers, in the form of energy savings, and for the electricity system in total, through the optimized use of the generation and network infrastructures, are important, the Energy Efficiency Directive (EED) 2012/27/EU of the European Parliament sets out the framework for encouraging market actors to facilitate DR. More specifically, DR should be recognized by the transmission and distribution network operators as a system resource that can participate, on equal terms with traditional supply resources, in all types of organized electricity markets (over-the-counter markets and electricity exchanges for trading energy, capacity, balancing and ancillary services in all timeframes, including forward, day-ahead and intra-day markets).

To this end, during 2014-2015 ENTSO-E amended the Network Code on Electricity Balancing to include demand side response as a provider of balancing services. After its adoption, as already recommended by ACER on July 2015, TSOs will have the opportunity to select from a broader range of balancing services providers (demand facility owners, aggregators, power generating facilities, owners of storage 
elements).

At the same time, the deployment of intelligent metering systems poses new challenges with regard to data protection, security, transparency and interoperability, with appropriate guidance given in Commission Recommendation 2012/148/EU. In order to ensure the protection of personal data, the European Commission developed the so-called Data Protection Impact Assessment Template for Smart Grid and Smart Metering Systems promoting a common methodology within the EU for guaranteeing adequate and harmonized processing of personal data by data controllers. After the twoyear test-phase of the Template is concluded (by the end of 2016), the enhanced document will serve as a guide for Smart Grid stakeholders to identify and assess privacy risks within the framework of the reformed Data Protection Regulation that is to replace Directive 95/46/EC.

\section{ASSESSMENT OF THE EUROPEAN REGULATORY FRAMEWORK}

Driven by the stipulations of the regulatory framework in the EU, several Member States have put in place appropriate legislation that typically enables the integration of DR in market operations. Despite this, the regulated financial model applied to the DSOs (who are regulated 'natural monopolies') in several EU countries does not include adequate incentives for them to be engaged in DR services.

Indeed, energy regulators in several EU countries do not recognize Smart Grid investments in the DSO's Regulatory Asset Base (RAB). In other member states, regulators exclude the benefits for all actors of the electricity value chain (consumers, suppliers, operators, etc.), in their Cost Benefit Analysis (CBA) for smart meters and Smart Grids. This approach neglects the required level of Smart Grid capital expenditures (CAPEX) for facilitating new services, especially in the automation and communication infrastructures. As a consequence DSOs are rather reluctant to invest in smartening their grids, investing instead in upgrading network assets.

Calculations made by EURELECTRIC that are based on figures of the International Energy Agency (IEA) [5], raise the investment needs for the European distribution grid to an amount of 480bn Euros up to 2035.

In the same report some of the major shortcomings of the current European regulatory frameworks are highlighted. In particular, it is mentioned that sub-optimal rates of return and regulatory instability hamper investment in smarter distribution grids; also that the roll-out of smart meters is delayed by lack of clarity regarding the roles and responsibilities of individual market players; and that regulators take a narrow view when evaluating cost efficiency, penalizing extra expenditure on research and development or pilot projects and encouraging business-as-usual expenditure instead. On the basis of the above, a key recommendation to the European Commission, European Regulators (ACER/ERGEG/CEER) and other stakeholders is that a fair rate of return is an essential requirement for Smart Grid investments. It is also suggested that these investments should be accepted in the $\mathrm{RAB}$, specifically requiring a shorter payback period.

Notable exceptions do exist in Europe however, which could serve as best practices for European regulation. In Italy for example, the energy regulator has launched a competitionbased procedure to incentivize selected Smart Grid/ DR projects by an extra $2 \%$ increase in weighted average cost of capital (WACC) for a period of 12 years. In the UK, an Innovation Funding Incentive was introduced in 2005 allowing up to $0.5 \%$ of annual revenue of DSOs to be spent on innovation. More recently, in 2010 the Low Carbon Networks Fund was set up to allocate $£ 500$ m over the period $2010-2015$ for trialing new DSO initiatives to prepare for smarter electricity networks.

\section{EUROPEAN STANDARDIZATION ASPECTS RELATED TO DEMAND RESPONSE}

The European Union supports an effective and coherent standardization framework, which ensures that standards are developed in a way that supports EU policies and competitiveness in the global market. Regulation 1025/2012 on European standardization sets the legal framework in which the different actors in the standardization system can operate. These actors are the European Commission, the European standardization organizations, industry, small and medium-sized enterprises (SMEs) and societal stakeholders.

The 2016 Rolling Plan on ICT Standardization [6] is the result of collaboration among major standardization stakeholders through the Multi-Stakeholder Platform on ICT Standardization. It covers all activities that can support standardization. Priority actions aim to ensure interoperability and facilitate ICT uptake in key areas. Among others the Plan offers details on the international contexts for the Smart Grid domain.

In this Plan the European Commission recognizes that standards are needed to cover the communication needs of the grid management, balancing and interfacing with the millions of new renewable sources, as well as standards for the complex interactions of the new distributed energy market, and in particular a transparent DR scheme. It is also recognized that DR initiatives will only fully deliver their potential, if they are complemented by a fully rolled out high speed open information and communication technology infrastructure.

The European Commission considers that the issue of DR related standardization is still open and towards this direction it established an Expert Group as part of its Smart Grid Task Force with the aim to assess the interoperability and standards used in the Smart Grid environment, with focus on the delivery of DR-ready interfaces. On parallel, it mandated the three European Standards Organizations (ESOs), namely CEN, CENELEC and ETSI to develop a framework that enables them to perform continuous standard enhancement and development in the Smart Grid field.

While this is work on progress, several European DR related initiatives are based on standards developed by the International Electrotechnical Commission (IEC). The standard IEC 62746 specifies the interfaces, communication 
protocol profiles and data models relevant for systems connected to the Smart Grid. In particular the IEC 62746-10-1 is dedicated to the communication protocol related to Demand Response. The standard is entitled IEC PAS - OpenADR for Demand Response. The code-name OpenADR pertains to a standard that was originally defined by the Lawrence Berkeley National Laboratory Demand Response Research Center as a low cost communication solution for the reliable, robust and cost-effective Demand Response communication. Therefore, the OpenADR 2.0b profile specification was approved by IEC as a Publicly Available Specification, which means that it serves as a basis for a new commission standard to be developed.

From CENELEC side it is recognized that within a Smart Grid, the AMI may also be used for Demand Response / Demand Side Management in connection with demand and production (generation) flexibility systems. In particular, interfaces where the Demand Response Management System utilizes the AMI as the channel to the home/building were identified under the M/441 mandate [7] as the H2 and H3 interfaces that support the provision of energy efficiency and demand-side management services.

Another EU backed standardization activity that is relevant to DR focuses on the definition of a new reference language for energy-related data (Smart Appliances Reference ontology, SAREF). This new language will allow devices in a home/building to seamlessly exchange information with any energy management system (which could physically be in the home or in the cloud). SAREF is now an ETSI standard, part of the machine-to-machine (M2M) architecture. If deployed, it will enable DR to flourish, will bring energy and cost savings for building owners and users, and will open new markets.

\section{Commercial Demand Response SERVICES IN EURope}

Europe's DR needs are not driven by summertime peak loads, as is the case in the United States. Europe does have some wintertime electric heating loads, but the bigger drivers are the system-wide effects of the continent's growing share of intermittent wind and solar power [8].

Theoretical calculations of the capacity of DR in the European market indicate that it could reach $14 \%$ of peak demand and an economic benefit that varies from few Euros/kw/year to higher amounts, depending on the presence of competing sources of flexibility in the system [9].

In practice, the penetration of DR services in Europe is still rather low. The vast majority of consumers do not have access to DR services within any market, to say nothing of the full range of balancing, reserves and wholesale markets [10]. In fact, as already mentioned in Sections II and III, Europe has only recently started establishing the framework that allows demand-side resources to participate.

While countries such as Belgium, Finland and Switzerland have reached a level where DR is a commercially viable, and countries such as Austria and Ireland are about to enable DR programs in the near future, Germany, Spain, Italy and Poland do not offer this potential nor is there legislation in place to enable it in future. This is correlated with the CAPEX issue analyzed in Section III, and a need for novel business models and retailer processes. In fact, even in countries where DR companies are established (e.g. Sweden, the Netherlands, Austria and Norway) there are still significant regulatory barriers that hinder market growth. This is usually due to requirements for participating in the markets that are not adjusted for both generation and demand-side resources.

The situation in the Nordics, Great Britain and France is rather positive: customers do have the opportunity to participate in implicit DR. In France, about $6 \mathrm{GW}$ of capacity is expected to be needed to fill in gaps that can't be met by the country's nuclear and fossil-fuel-fired generator fleet, and this is a share that could potentially be addressed by aggregated DR assets [8]. In UK the launching of the GB Capacity Market has not yet created a level playing field for DR to participate. As a matter of fact, the majority of the $45 \mathrm{GW}$ of winning bids in the first capacity auction in December 2015 went to power production instead of DR. In the remainder of European Member States aggregated DR is either illegal or its development is seriously hindered for all market participants large industrial sites, suppliers or independent aggregators- by regulatory barriers [10].

\section{ONGOING RESEARCH ACTIVITIES RELATED TO DEMAND RESPONSE}

The DR-related research activities focus on developing new methodologies, ICT tools and services that can enable integration of the DR in the energy market, while facilitating customer engagement and supporting security and privacy. A large domain of exploration is related to tools and methodologies for DR forecasting, load forecasting, customers' profiling and segmenting, scheduling and dispatching DR events, as well as measuring, verifying and evaluating the results of DR participations. A significant amount of work has been realized in this domain in the past period (e.g. [11]) and currently the focus is shifting on validating those tools and methodologies in real-life, largescale pilots, aiming towards the delivery of market-ready products. As part of this trend, several large-scale pilot projects have been and are being deployed, for exploring the feasibility of DR in residential and commercial customers.

According to the Joint Research Center of the European Commission, which maintains an inventory of Smart Grid projects across the European Union, for the period of 2002 to 2014 , over 450 research \& development and demonstration \& deployment projects have been active in the domain of Smart Grids, a large amount of which concerned DR and active consumer participation, with a total investment of over $3 \mathrm{bn}$ euro [12]. Projects of the recent past studied the concept of implicit DR for reducing peak load and providing local grid services and explored the replication and scalability potential of such initiatives. Among relevant ongoing activities we refer indicatively the SmarterEMC2 project in which DR is mainly considered from the viewpoint of the DSOs, as a means to achieve network stability, and NobelGrid project in which a solution that enables other stakeholders (ESCOs, DR Aggregators and Energy Retailers) to handle the flexibility of 
DR-related assets in the energy markets is implemented.

The successful incorporation of DR in the energy markets imposes the need for development of new business models and the participation of new business actors (e.g. DR Aggregators, Retailers, ESCOs), whilst also allows the emergence of new services (e.g. ancillary services in the distribution grid). The inquiry on how these new services can be supported by current market design, as well as the clarification of the new roles, responsibilities and relevant interactions of the various actors participating in the market (e.g. DSOs, TSO, BRPs, Retailers, Aggregators) are investigated. A transparent operation of the market is an active subject in the industry, while in parallel, several research and innovation initiatives aim at proposing new market design approaches to accommodate Demand Response services.

Another domain of exploration relevant to DR is its integration into Customer Energy Management Systems (CEMS), with the aim to optimize at the building level energy consumption, production and storage, while taking into account the availability and cost of energy supplied via the distribution grid. Such systems, as well as smart home devices, have in most cases limited interoperability and are often linked to a specific vendor, technology or standard. Achieving full interoperability between products and systems and seamlessly integrating all required components of a CEMS shall provide a significant boost towards wider adoption of such services. European Commission's initiatives such as the promotion of SAREF ontology or the DR-relevant research topics defined in the context of Horizon 2020 framework act as facilitators of this intention.

Finally, the transformation of consumers to prosumers imposes significant research challenges in terms of engagement and privacy. In this particular domain, research focus is now shifted to issues such as the understanding the consumer's active role and behavior [13] and the psychology of consumer engagement in DR [14]. Another significant research objective is the design of successful strategies with attractive incentives or reward schemas, as well as effective ways of communicating with consumers (e.g. in-home displays, social competition / comparison), with the aim to motivate them to actively participate in DR initiatives. Last but not least, the issue of privacy and security is addressed also in the context of DR-relevant projects.

\section{CONCLUSION}

A critical assessment of the current status of DR in Europe reveals that despite the intensive regulatory and policy formulation processes followed by various EU institutions, and despite the distinct cases where DR is a functioning tool of the markets, we are still far from a wide rollout and adoption of relevant services. Also, it is evident that the potential of DR varies significantly among EU countries, as a consequence of the significantly different conditions in terms of energy production portfolio, the energy market liberalization, the complexity of the business models, and the fragmentation and heterogeneity of the regulatory frameworks at national level. Notwithstanding the potential benefits of DR for the consumers, the grid and the involved business actors, a systematic cost benefit analysis will be required to determine the soundness of DR among EU Member States, while on parallel, further homogenization and standardization of the regulatory framework will be needed to facilitate the establishment of sustainable business models around DR.

\section{ACKNOWLEDGMENT}

This work was supported in part by the European Community's Horizon 2020 Framework Program under grant agreement No 646470, SmarterEMC2 Project. The authors would like to thank their colleagues in SmarterEMC2 project for their collaboration.

\section{REFERENCES}

[1] ENTSO-E. (2015, March). Towards smarter grids: Developing TSO and DSO roles and interactions for the benefit of consumers, Brussels, Belgium. [Online]. Available: https://www.entsoe.eu/Documents/Publications//Position papers and reports/150303_ENTSO-E_Position_Paper_TSO-DSO_interaction.pdf. Accessed on: July 06, 2016.

[2] Smart Grid Task Force Expert Group 3. (2015, January). Regulatory recommendations for the deployment of flexibility. Brussels, Belgium. [Online]. Available: http://ec.europa.eu/energy/sites/ener/files/ documents/EG3\%20Final\%20-\%20January\%202015.pdf. Accessed on: July 06, 2016.

[3] Smart Energy Demand Coalition. (2015, July). Demand response: Clarification of the standard processes required between BRPs and independent aggregators. Ixelles, Belgium. [Online]. Available: http://www.smartenergydemand.eu/wp-content/uploads/2015/07/SEDCStandard-processes-required-between-BRPs-and-independentaggregators.pdf. Accessed on: July 06, 2016.

[4] Directive 2009/72/EU of the European Parliament and of the Council on energy efficiency. Official journal of the European Union. [Online] L211, 13 July 2009, p.55. Available: http://eur-lex.europa.eu/legalcontent/EN/TXT/?uri=celex:32009L0072. Accessed on: July 06, 2016.

[5] Eurelectric. (2011, February). Regulation for smart grids. Brussels, Belgium. [Online]. Available: http://www.eurelectric.org/media/25920/eurelectric_report_on_reg_for _sg_final-2011-030-0131-01-e.pdf. Accessed on: July 06, 2016.

[6] European Commission. (2016, February). Rolling plan on ICT standardisation. [Online]. Available: http://ec.europa.eu/newsroom/dae/document.cfm?doc_id=14510. Accessed on: July 06, 2016.

[7] European Commission. (2012, March). M/441 Standardisation mandate to CEN, CENELEC and ETSI in the field of measuring instruments for the development of an open architecture for utility meters involving communication protocols enabling interoperability. [Online]. Available: http://ec.europa.eu/growth/tools-databases/mandates/index.cfm? fuseaction=search.detail\&id=421\#. Accessed on: July 06, 2016.

[8] Jeff St. John, "Demand response markets in Europe begin to blossom", Greentech Media, 08 May 2015. [Online]. Available: www.greentechmedia.com/articles/read/restore-raises- $7 \mathrm{~m}$-for-europesgrowing-demand-response-markets. Accessed on: July 06, 2016.

[9] Cambridge Economic Policy Associates Ltd, TPA Solutions \& Imperial College London. (2014, September). Demand side flexibility: The potential benefits and state of play in the European Union. [Online]. Available:

http://www.acer.europa.eu/official_documents/acts_of_the_agency/refer ences/dsf_final_report.pdf. Accessed on: July 06, 2016.

[10] Smart Energy Demand Coalition. (2015, February). Enabling independent aggregation in the European electricity markets. Ixelles, Belgium. [Online]. Available: http://smartenergydemand.eu/wpcontent/uploads/2015/02/SEDC-Enabling-Independent-Aggregation.pdf. Accessed on: July 06, 2016.

[11] P. Koponen, J. Ikäheimo, A. Vicino, A. Agnetis, G. De Pascale, N. R. Carames, J. Joseba, E. F. Sánchez-Úbeda, P. Garcia-Gonzalez, and R. Cossent, "Toolbox for aggregator of flexible demand," Energy 
Conference and Exhibition (ENERGYCON), 2012 IEEE International, Florence, 2012, pp. 623-628.

[12] C. F. Covrig, M. Ardelean, J. Vasiljevska, A. Mengolini, G. Fulli, E. Amoiralis, M. Sanchez Jimenez, C. Filiou (2014). Smart grids projects outlook 2014. Joint research center science and policy reports. Luxemburg: European Commission [Online]. Available: http://ses.jrc.ec.europa.eu/sites/ses.jrc.ec.europa.eu/files/u24/2014/report /ld-na-26609-en-n_smart_grid_projects_outlook_2014_-_online.pdf. Accessed on: July 06, 2016.

[13] F. Gangale, A. Mengolini, I. Onyeji, "Consumer engagement: An insight from smart grid projects in Europe" in Energy Policy vol. 60 no. September 2013 p. 621-628.

[14] J. Stromback, C. Dromacque, and M. Jassin. (2011). The potential of smart meter enabled programs to increase energy and system efficiency: A mass pilot comparison. VaasaETT. [Online]. Available: http://esmig.eu/sites/default/files/2011.10.12 empower_demand_report final.pdf. Accessed on: July 06, 2016.

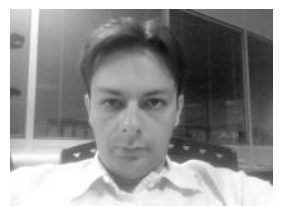

Ilias E. Lamprinos was born in Lesvos, Greece, in 1977. He received the diploma in Electrical and Computer Engineering in 2000 and his $\mathrm{PhD}$ degree in Electrical and Computer Engineering in 2006, both from the National Technical University of Athens (NTUA), Greece.

He is with Intracom Telecom in Athens, Greece since 2007, dealing with research and innovation activities that focus on the end-to-end integration of IT systems in diverse domains (smart energy, smart agriculture, health). He has published several papers in peer reviewed journals and conferences and served as reviewer for scientific journals and conferences. Dr Lamprinos is currently the Project Coordinator of the H2020LCE07-2014 project SmarterEMC2: Empowering SG Market ACtors through Information \& Communication Technologies.

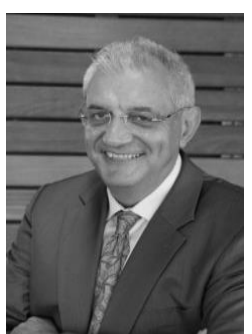

Nikos D. Hatziargyriou is Chairman and CEO of the Hellenic Distribution Network Operator. Since 1984 he is with the Power Division of the Electrical and Computer Engineering Department of the National Technical University of Athens and since 1995 he is full professor in Power Systems. From February 2007 to September 2012, he was Deputy CEO of the Public Power Corporation (PPC) of Greece, responsible for Transmission and Distribution Networks, island DNO and the Center of Testing, Research and Prototyping. He is Fellow Member of IEEE, past Chair of the Power System Dynamic Performance Committee, Distinguished member of CIGRE and past Chair of CIGRE SC C6 "Distribution Systems and Distributed Generation". He is chair of the Advisory Council of the EU Technology Platform on SmartGrids. He is member of the Energy Committee of the Athens Academy of Science. He has participated in more than 60 R\&DD Projects funded by the EC and the industry, and has coordinated among others, the EU "CARE", "MORE CARE", "MERGE", "Microgrids" and "More Microgrids" projects. He is author of the book "Microgrids: Architectures and Control" and of more than 200 journal publications and 500 conference proceedings papers.

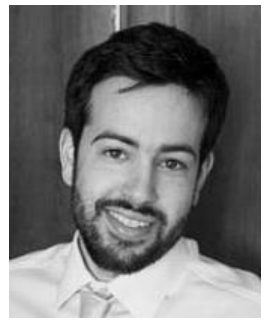

Isidoros E. Kokos was born in Heraklion, Greece, in 1985. He received his Dipl.Eng. degree in electrical and computer engineering from National Technical University, Athens, Greece, in 2008. He obtained an M.Eng. degree in the post graduate course of Energy Production \& Management from the same institution, in 2010.

$\mathrm{He}$ has working experience as a Software Engineer in Intralot, during 2009 to 2010. From 2011 to 2014 he worked as an Analyst and Consultant in the startup company Intelen, working in the development of ICT services in the energy domain, whilst also participated in R\&D projects founded by EU commission. In 2015 he joined Intracom Telecom in Peania, Greece, as an R\&D Engineer and is actively engaged in projects focused in the Smart Grids domain.

Mr. Kokos is a member of the Technical Chamber of Greece (TEE).

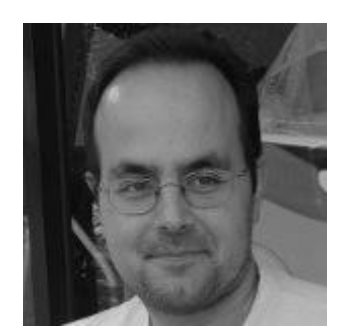

Aris L. Dimeas was born in Athens, Greece, in 1977. He received the diploma in Electrical and Computer Engineering as well his $\mathrm{PhD}$ degree from National Technical University of Athens (NTUA). He is currently a researcher at Electrical and Computers Engineering Department of NTUA. He has experience in a variety of applications related to Power Systems operations, Renewable Energy Sources, Artificial Intelligence on Power Systems, Smart Grids and Control Software development. His specific experience also includes development of control software for Demand Side Management and communication interfaces between Energy Management Systems and SCADAs. Furthermore he was a consultant (2007-2012) in the department of Non Interconnected Islands of the Greek Public Power Corporation (PPC). He was involved with the preparation of the code for energy market in Crete and also developed simulations of System Operation focusing on the concept of Hybrid Stations. Additionally, he is in collaboration with other sectors/departments of PPC/HEDNO dealing with electronic meters, intelligent networks and research programs. He is member of the Technical Chamber of Greece and of IEEE. 